\title{
An Analysis of HST/FOS Spectra of Spatially Resolved Compact OB Systems in the LMC
}

\author{
E. Niemczura \& H. Cugier \\ Astronomical Institute of the Wroclaw University, ul. Kopernika 11, \\ 51-622 Wroclaw, Poland
}

\begin{abstract}
An analysis of the spatially resolved (0".1) main-sequence stars of four compact multiple systems located in the Large Magellanic Cloud (LMC) is given. For this purpose we compared theoretical synthetic spectra with the observations obtained by means of the Faint Object Spectrograph (FOS) aboard the Hubble Space Telescope (HST).
\end{abstract}

\section{Introduction}

Spectroscopic observations obtained with the high spatial resolution have the important meaning for study multiple stellar systems in the Magellanic Clouds, cf. e.g., Massey and Hunter (1998) and Walborn et al. (1999).

In this paper we examined archival spectroscopic data obtained with the HST Faint Object Spectrograph (HST/FOS). Uncontaminated spectra of the components of compact OB systems located in LMC enable us to study their basic parameters, like effective temperatures (Teff), surface gravities $(\log g)$, stellar rotational velocities $(\mathrm{V} \sin i)$ and radial velocities $\left(\mathrm{V}_{\mathrm{rad}}\right)$.

\section{Observations and their analysis}

We analysed spectra of the stars from Breysacher 73 in 30 Doradus B, NGC 2044 in 30 Doradus C, HD 32228 in the shell H II region Henize N11 and OB group of Lucke-Hodge 10 in the nebula of the N11 shell. The observations were obtained in the wavelength range of $3240-4780 \AA$ with the spectral resolution of about $3 \AA$ collected by means of FOS with 0 ". 3 aperture. The typical signal-to-noise ratios of these spectra are $\mathrm{S} / \mathrm{N} \approx 50-70$, except of the objects in NGC2044, for which $\mathrm{S} / \mathrm{N} \approx 20-25$.

These observations were compared with theoretical spectra. For this purpose we adopted the grid of non-LTE models calculated by Gonzales Delgado and Leitherer (1999). They used the TLUSTY code of Hubeny (1988) for $\mathrm{T}_{\text {eff }}>25000 \mathrm{~K}$ and Kurucz's (1993) LTE models for cooler stars.

\section{Results and discussion}

We selected all objects without strong emission lines for further analysis due to the steady-state photospheric models used. Figure 1 illustrates the best-fit 

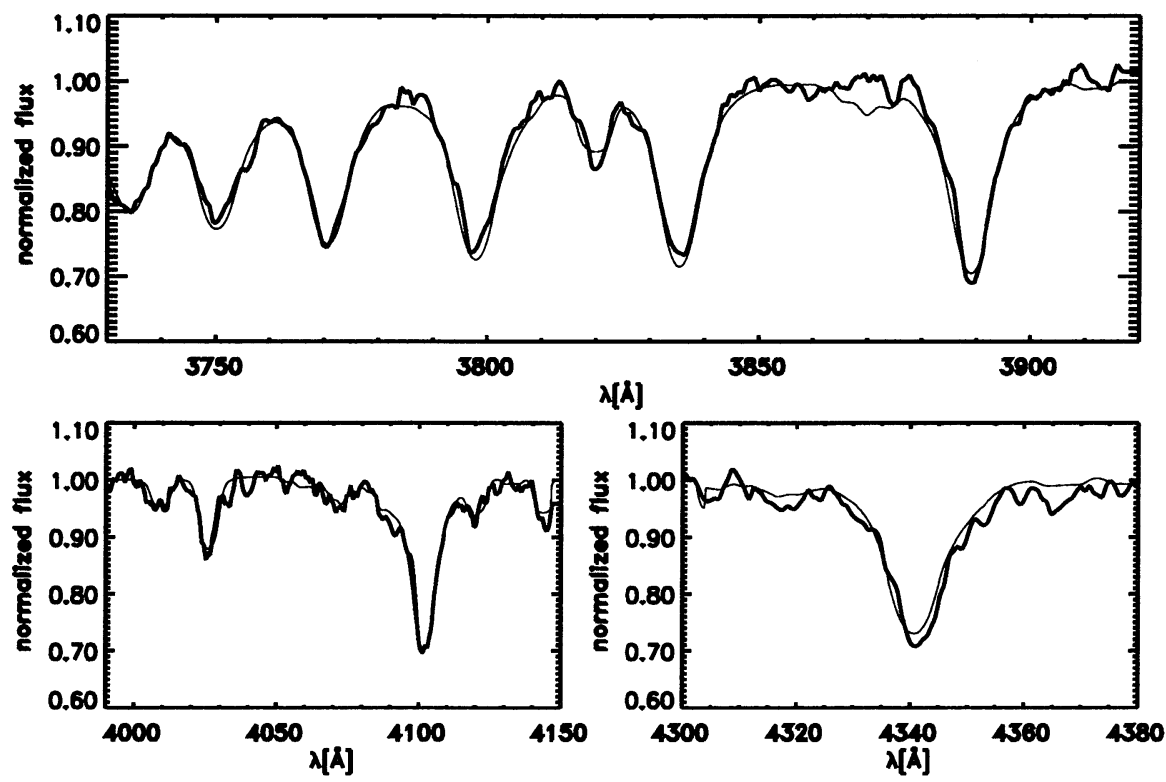

Figure 1. HST/FOS spectrum of the H-M f component in HD32228 plotted together with the theoretical one calculated for $\mathrm{T}_{\text {eff }}=30000 \mathrm{~K}$ and $\log g=4.0$.

solution for H-M f/HD32228, which can be regarded as a characteristic one for the program stars. In general our values of $\mathrm{T}_{\text {eff }}$ are lower then those given by Walborn at al. (1999). The differences can reach $5000 \mathrm{~K}$. The surface gravities are in the range of 4.0-4.5 dex. in an agreement with the fact that we analysed "normal" stars of the luminosity class V. All analysed stars seems to be fast rotators with Vsin $i \geq 160 \mathrm{~km} / \mathrm{s}$. The measured radial velocities of the order of $200-280 \mathrm{~km} / \mathrm{s}$ probably reflect kinematic properties of the stellar systems. More details will be given in a future paper.

\section{References}

Gonzales Delgado, R. M., Leitherer, C. 1999, ApJ Suppl. 125, 479

Hubeny, I. 1988 in Comput. Phys. Commun. 52, 103

Kurucz, R.L. 1993, CD-ROM No. 13

Massey, P., Hunter D.A. 1998, ApJ 493, 180

Walborn, N.R., Drissen, L., Parker, J.Wm., Saha, A., McKenty, J.W., White, R.L. 1999, AJ 118, 1699 